\title{
GLUEBALLS AND TOPOLOGICAL CHARGE IN THE PRESENCE OF DYNAMICAL QUARKS ${ }^{\dagger}$ CONF-901072-.5
}

Khalil M. BITAR ${ }^{1}$, Thomas A. DEGRAND ${ }^{2}$, R. EDWARDS ${ }^{1}$, Steven GOTTLIEB ${ }^{3}$, U. M. HELLER ${ }^{1}$,

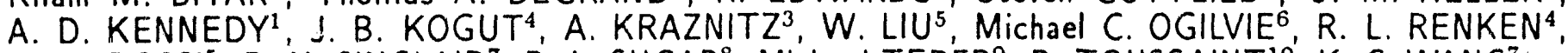
Pietro ROSSI ${ }^{5}$, D. K. SINCLAIR ${ }^{7}$, R. L. SUGAR ${ }^{8}$, Michael TEPER ${ }^{9}$, D. TOUSSAINT ${ }^{10}$, K. C. WANG ${ }^{7 *}$

(1) SCRI, The Florida State University, Tallahassee, FL 32306-4052, USA

(2) Physics Department, University of Colorado, Boulder, CO 80309, USA

(3) Department of Physics, Indiana University, Bloomington, IN 47405, USA

ANL-HEP-CP- $-90-120$

(4) Department of Physics, University of Illinois, Urbana, IL 61801, USA

(5) Thinking Machines Corporation, Cambridge, Mass. 02142, USA

(6) Department of Physics, Washington University, St. Louis, MO 63130 USA

(7) HEP Division, Argonne National Laboratory, Argonne, IL 60439, USA

DE91. 006459

(8) Dept. of Physics, University of California, Santa Barbara, CA 93106 USA

(9) Dept. of Theoretical Physics, University of Oxford, Oxford OX1 3NP UK

(10) Depa.tment of Physics, University of Arizona, Tucson, AZ 85721 USA

We have estimated the string tension and the masses of the $0^{++}$and $2^{++}$glueballs in lattice QCD with 2 light flavours of staggered quarks. In addition we have measured the topological charge and hence susceptibility of our gauge field configurations. The simulations were performed at $6 / \mathrm{g}^{2}=5.6$ and quark masses $\mathrm{m}=0.01$ and 0.025 (lattice units) on lattice sizes ranging from $12^{4}$ to $16^{4}$.

Lattice $Q C D$. nables one to calculate the hadron mass spect $\cdot$ ' $m$ from first principles, testing $Q C D$ by giving masses of known hadrons, possibly predicting states which have yet to be seen, and clarifying the quark model assignments of poorly understood resonances. Glueballs, colourless bound states of gluons, are specific to QCD and not predicted by simple quark models. Thus predicting whether $Q C D$ does in fact require glueballs narrow enough to be seen, and if so calculating their masses to compare with experiment, promises to be a definitive test of QCD. We preser:; an exploratory calculation of the glueball masses, including the effects of dy-

*Presented by D.K. Sinclair.

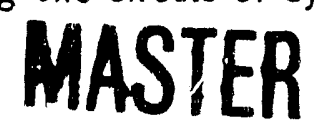

namical quarks which, by allowing glueball decay and glueball/meson mixing could completely change the spectrum observed in pure gauge theory.

The topological structure of the gauge fields of QCD is linked through the anomaly with the chiral behaviour of QCD. In particular it explains why there is no light isosinglet pseudoscalar meson, and explains the peculiar properties of the $\eta$ and $\eta^{\prime}$. An understanding of topological charge is necessary to completely understand confinement and chiral symmetry breaking. Since topological fluctuations are sensitive to the presence of light quarks it is helpful to know how they depend on quark mass. We present measurements of the dependence of topological susceptibility on quark mass.

tSupported by DOE contracts DE-FG02-85ER-40213, DE-AC02-86ER-40253, DE-AC02-84ER-40125, DEASO3-81ER-40029, W-31-109-ENG-38 and DE-FC05-85ER250000 and NSF grants NSF-PHY87-01775, NSFPHY86-14185, NSF-PHY89-04035. ETA-10 and CM-2 CPU time at SCRI was made available under a DOE "Grand Challenge" grant. 
Our simulations, performed using the hybrid molecular dynamics algorithm to incorporate 2 light degenerate flavours of "noisy" staggered quarks have been discussed elsewhere ${ }^{1,2}$. All simulations were run at $\beta=6 / g^{2}=5.6$. The measurements of glueball masses and topological charge used 500 configurations on a $12^{4}$ lattice at quark mass $m=0.025$, 500 on a $12^{4}$ lattice at $m=0.01,100$ on a $12^{3} \times 24$ lattice at $m=0.01$ and 125 on a $16^{4}$ lattice with $m=0.01$. We are now running on a $16^{3} \times 32$ lattice with $m=0.01$.

Let us now describe our glueball mass calculations. We have used the "fuzzy" glueball wave functions of Teper $^{3}$ to improve the overlap with low lying glueballs, enhance the signal/noise ratio and allow the extraction of glueball masses from propagators at relatively small time separations. In this scheme we first define "blocked" or "iuzzy" links recursively, viz.,

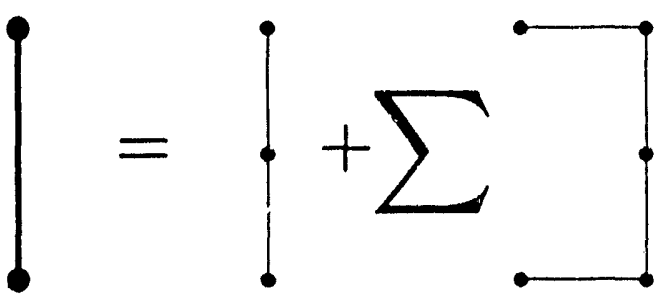

where the sum is over the 4 spatial directions orthogonal to the link we are blocking. The result is projected back on to SU(3). We consider blocking levels 0 (unblocked), 1, 2, 3. These blocked links are used to create wave functions for a given template (see references ${ }^{4,5}$ for templates), and spin/parity assignment. If $W(T)$ is such a wave function with zero spatial momentum, restricted to the hyperplane at time $T$, our zero momentum correlation function is defined by

$$
C(T)=\frac{1}{v} \sum_{t}<W^{\dagger}(t) W(t+T)>
$$

As in the quenched case ${ }^{4}$ blocking does greatly enhance the signal/noise ratio as seen in Figure 1.

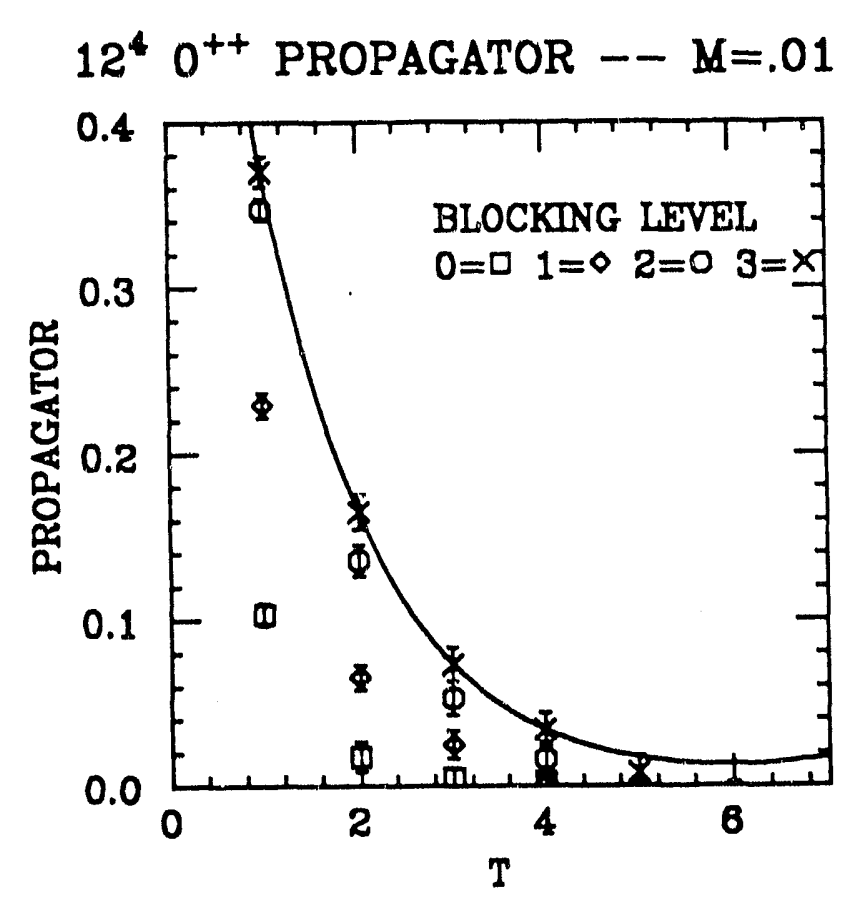

FIGURE 1. The $0^{++}$propagators for different blocking levels with wave functions based on the simple plaquette for a $12^{4}$ lattice with $m=0.01$. The fit is to the form $0.8298[\exp (-0.8080 T)+\exp (-0.8080(12$ $T)$ )] which fits the points $T=1$ and $T=2$ of the blocking level 3 propagator exactly.

The effective mass is extracted from (2) using the asymptotic form

$$
C(T) \sim A\left[e^{-M T}+e^{-M\left(N_{t}-T\right)}\right]
$$

(where $M$ is the lowest mass state for the chosen wave function) to fit $C(T)$ and $C(T-1)$. At large $T$ the effective mass should approach $M$. Figure 2 shows the effective mass plot for the blocking level 3 propagator for the simple plaquette wave function on the $12^{4}$ lattice at $m=0.01$. Notice that the errors increase rapidly past $T=2$, a property of all propagators we have calculated, which forces us to use the $T=2$ effective masses (from fitting $C(2) / C(1)$ to (3)) as our estimate for the value of the lightest masses in the appropriate channels. These are really only upper bounds. We appeal to the quenched case ${ }^{4}$ with its higher statistics to indicate that these might well be reasonable estimates to the actual glueball masses, provided mixing of glueball and meson states is small. Our limited evidence that this mixing is indeed small will be presented in a forthcoming paper ${ }^{5}$. 
$12^{4} 0^{++}$EFFECTIVE MASSES - M $=.01$

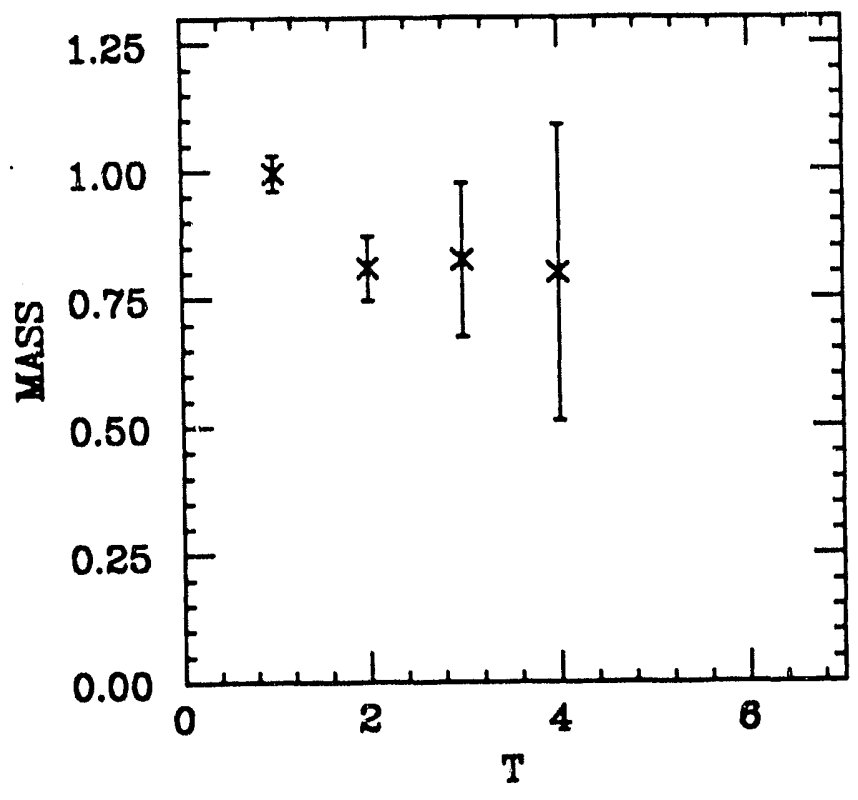

FIGURE 2. The effective mass for the blocking level 3 single plaquette $0^{++}$wave function on a lattice at $m=0.01$ as a function of $T$.

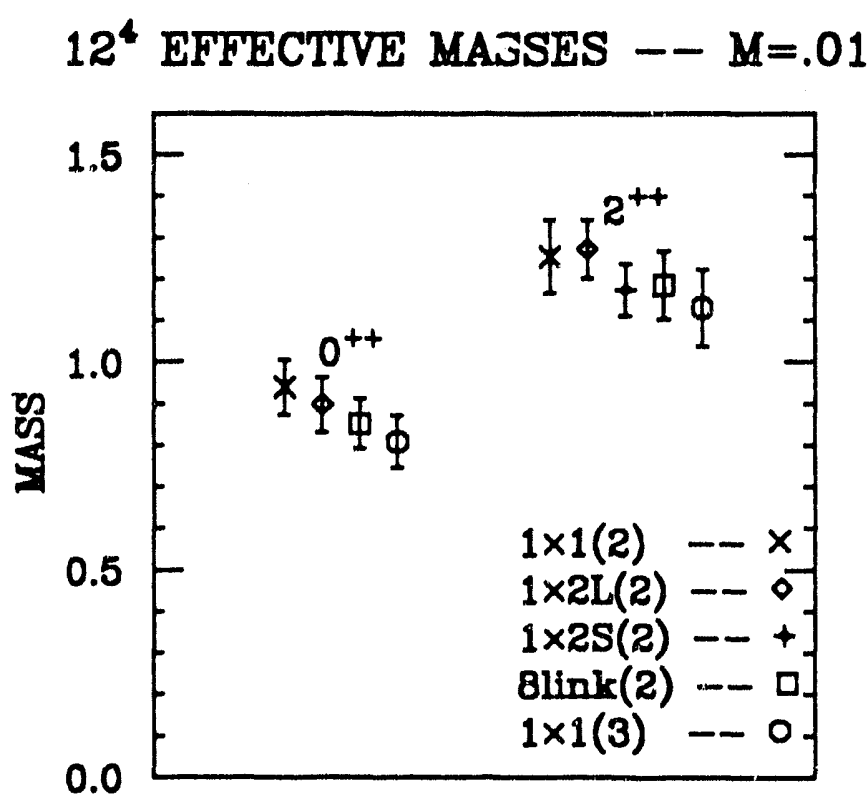

FIGURE 3. Effective masses at $T=2$ for various wave functions on a $12^{4}$ lattice with $m=0.01$.

Here we were only able to extract a decent signal for the 2 lowest lying glueball states, the $0^{++}$ and $2^{++}$states, and for the blocked Wilson/Polyakov line, whose mass per unit length is $\kappa$ (the string ten- sion). Figure 3 gives the $\mathrm{0}^{++}$and $2^{++}$mass estimates for various templates/blocking levels. For definition of these wave functions see references ${ }^{4,5}$.

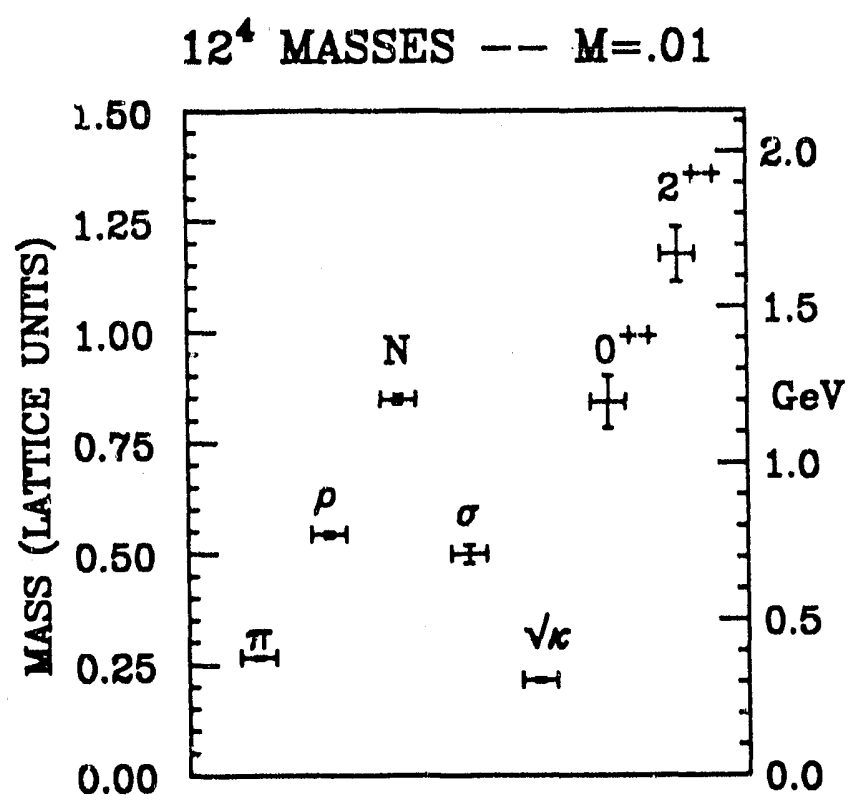

FIGURE 4. Glueball and hadron masses on a $12^{4}$ lattice at $m=0.01$

Finally, Figure 4 shows the glueball masses compared with the meson and nucleon masses calculated from the same gauge configurations. The $\mathrm{GeV}$ scale comes from arbitrarily taking the rho mass at $m=0.01$ to have its experimental value $(770 \mathrm{MeV})$. This implies a $0^{++}$glueball mass of around $1.2 \mathrm{GeV}$ and a $2^{++}$mass of around $1.7 \mathrm{GeV}$, while $\sqrt{\kappa} \sim$ $300 \mathrm{MeV}$. Although the value of $\sqrt{\kappa}$ is somewhat lower than the favoured $420 \mathrm{MeV}$, on the $16^{4}$ lattice this number is around $370 \mathrm{Mev}$, indicating that the depressed value could well be a finite size effect.

Now let us discuss topological charge measurements. The topological charge of each configuration was estimated using the cooling method ${ }^{6}$. Here the gauge fields were cooled using a Cabbibo-Marinari heat bath with the pure gauge action to smooth the field. The topological charge $Q$ of the cooled gauge configuration, which should represent the topological charge of large instantons in the uncooled configuration was calculated as

$$
Q=\frac{1}{32 \pi^{2}} \sum_{\text {sites }} \epsilon_{\mu \nu \rho \sigma} \operatorname{Tr}\left(U_{\mu \nu} U_{\rho \sigma}\right)
$$


where $U_{\mu \nu}$ is the product of the gauge fields $U$ around a plaquette in the $\mu \nu$ plane. Each $Q$ was rounded to the nearest integer. Figure 5 shows that this is justified.

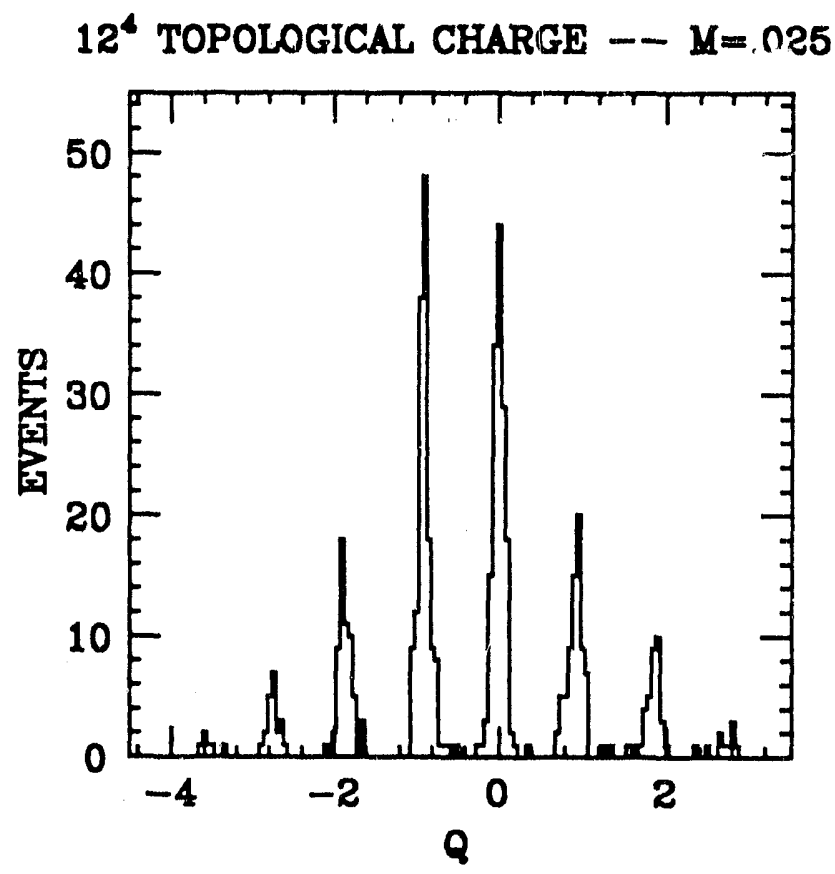

FIGURE 5. Histogram of the raw topological charge distribution on a $12^{4}$ lattice at $m=0.025$.

From $Q$ we obtained the topological susceptibility

$$
x=<Q^{2}>/ V
$$

For the confined phase we expect ${ }^{7}$

$$
\chi \propto m
$$

for small $\mathrm{m}$ ( $\chi \propto m^{n_{f}}$ in the unconfined phase). Instead we find

$$
\chi \propto m^{0.41(23)}
$$

Whether this is due to long time correlations leading to gross underestimates of the errors (a scenario for which we have some evidence), or whether it is due to other reasons (such as being too far from the continuum), only time will tell.

For SU(2) gauge theory with 4 flavours of staggered fermions it has been found that ${ }^{7}$

$$
\chi \propto m^{1.36(23)}
$$

in the confined region and is consistent with $\mathrm{m}^{4}$ in the deconfined region.

In the future we intend to improve statistics and perform a full variational calculation of glueball masses. In addition we plan to measure glueball$q \bar{q}$ mixing directly. More measurements of the mass dependence of $\chi$ in both QCD phases are planned.

\section{REFERENCES}

1. K. M. Bitar, T. A. DeGrand, R. Edwards, S. Gottlieb, U. M. Heller, A. D. Kennedy, J. B. Kogut, A. Krasnitz, W. Liu, M. C. Ogilvie, R. L. Renken, P. Rossi, D. K. Sinclair, R. L. Sugar, M. Teper, D. Toussaint and K. C. Wang, Florida State University preprint FSU-SCRI-90-98; Phys. Rev. Lett. 65, 2106 (1990).

2. Talks presented by T. A. DeGrand and $D$. Toussaint at this conference.

3. M. Teper, Phys. Lett. 183B, 345 (1987).

4. C. Michael and M. Teper, Nucl. Phys. B314, 347 (1989).

5. The HEMCGC collaboration (in preparation).

6. M. Teper, Phys. Rev. Lett. 162B, 357 (1985); J. Hoek, M. Teper, J. Waterhouse, Nucl. Phys. B288, 589 (1987).

7. J. B. Kogut, D. K. Sinclair and M. Teper, Oxford University preprint OUTP-90-14P (1990) 


\section{DISCLAIMER}

This report was prepared as an account of work sponsored by an agency of the United States Government. Neither the United States Government nor any agency thereof, nor any of their employees, makes any warranty, express or implied, or assumes any legal liability or responsibility for the accuracy, completeness, or usefulness of any information, apparatus, product, or process disclosed, or represents that its use would not infringe privately owned rights. Reference herein to any specific commercial product, process, or service by trade name, trademark, manufacturer, or otherwise does not necessarily constitute or imply its endorsement, recom. mendatior, or favoring by the United States Government or any agency thereof. The views and opinions of authors expressed herein do not necessarily state or reflect those of the United States Government or any agency thereof. 

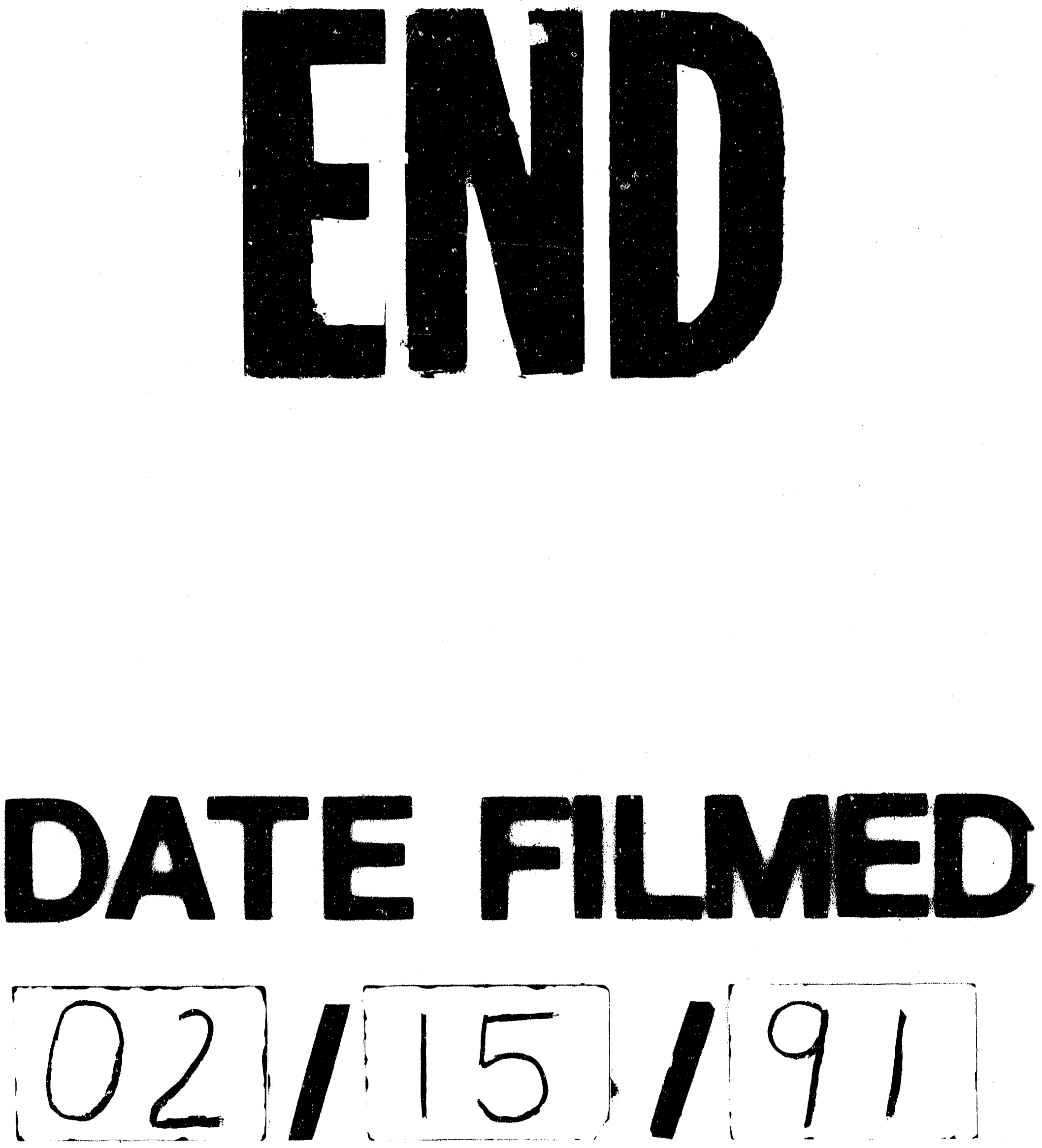


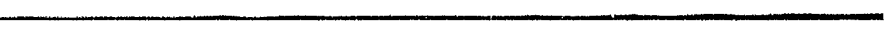

